\title{
Mercado de trabajo en Biblioteconomía y Documentación. Estudios sobre la inserción laboral de los titulados universitarios
}

\author{
Por Carlos Tejada Artigas y José Antonio Moreiro
}

\begin{abstract}
Resumen: Se describen los estudios realizados en el seguimiento laboral de los titulados universitarios en biblioteconomía y documentación en España. Estos análisis definen ciertas características del mercado de trabajo al que acceden estos graduados, tales como su volumen y dinamismo pero también sus condiciones, bastante precarias. Además, a pesar de la importancia del sector público, el sector privado cada vez emplea más a los jóvenes profesionales. De la misma manera el empleo en centros de documentación crece de forma porcentual más que en los archivos y bibliotecas. Este tipo de análisis se debe tener en cuenta en la concepción de los programas académicos de las universidades.
\end{abstract}

Palabras clave: Graduados de las escuelas de biblioteconomía, Inserción laboral, Mercado de trabajo, Información y documentación,

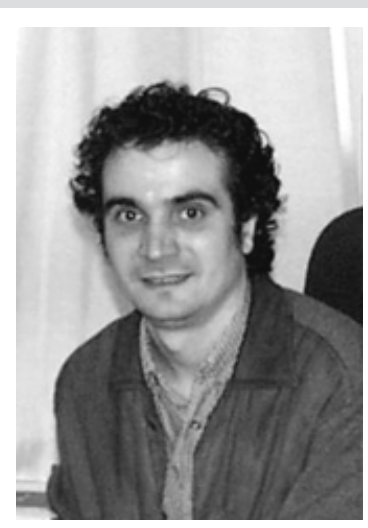
Carlos Tejada Artigas,
profesor y subdirector
de la Escuela Universita-
ria de Biblioteconomía y
Documentación de la
Universidad Compluten-
se de Madrid. Doctor en
documentación por la
Universidad Carlos III
de Madrid. Miembro de
la Junta Directiva y de la
Comisión de Certifica-
ción de Sedic. Universidad Carlos III de Madrid, Universidad Complutense de Madrid.

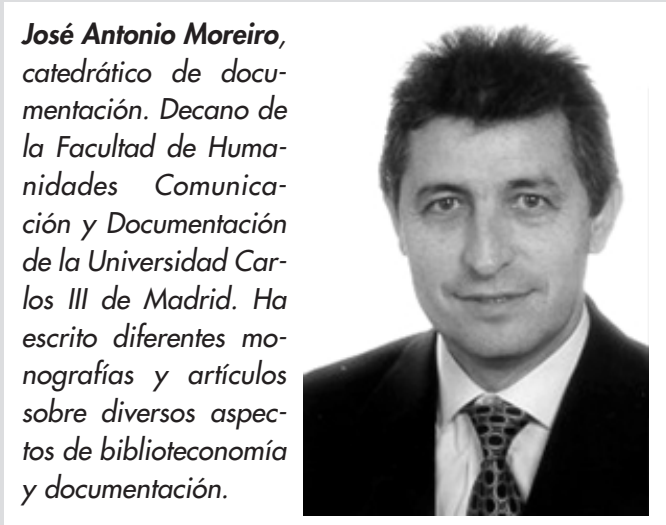

Title: The labour market for LIS graduates: studies on entry into the workforce

Abstract: This article describes the work done in monitoring the careers of LIS (library and information sciences) graduates in Spain. These analyses define some of the features of the labour market which the graduates gain access to, such as its volume and dynamism but also relatively quite poor conditions. Despite the importance of the public sector, the private sector increasingly employs young professionals. Equally, job opportunities grow more in percentage terms in information centres than in archives and libraries. This kind of analysis must be taken into account by universities when designing their LIS curricula.

Keywords: LIS graduates, Employment prospects, Labour market, LIS, Universidad Carlos III de Madrid, Universidad Complutense de Madrid, Librarians and information scientists.

Tejada Artigas, Carlos; Moreiro, José Antonio. "Mercado de trabajo en Biblioteconomía y Documentación. Estudios sobre la inserción laboral de los titulados universitarios". En: El profesional de la información, 2003, enero-febrero, v. 12, n. 1, pp. 4-9.

\section{Introducción}

Un acercamiento a la situación del mercado de trabajo en información y documentación lo constituyen sin duda los estudios realizados desde las universidades sobre el seguimiento de los titulados en estas disciplinas. Estos informes, entre otros objetivos, intentan medir el grado de adecuación de la formación univer- sitaria al mundo laboral y profesional. En este artículo no nos vamos a ocupar de este aspecto que daría lugar a otro enfoque y desarrollo; tan sólo pretendemos recopilar estos estudios de inserción laboral y ofrecer un panorama del mercado de trabajo al que se enfrentan los profesionales más jóvenes en biblioteconomía y documentación. 


\section{Estudios sobre el seguimiento de la inserción laboral de los graduados en biblioteconomía y documentación a nivel internacional}

Son numerosos los llevados a cabo en todo el mundo sobre el tema y al ver algunos datos que ofrecen se puede concluir que la situación en España no difiere de las características de ese mercado de trabajo a nivel internacional. A continuación citamos algunos de los más recientes.

Marcella y Baxter (1998) realizaron el seguimiento de las 3 primeras promociones (1992/31995/6) del diploma de postgrado/máster de análisis de información de la Escuela de Información y Medios, de la Universidad Robert Gordon. Se utilizó para ello un cuestionario que obtuvo una tasa de respuesta del $45 \%$. Como conclusión se señalaba que estos titulados obtienen trabajo en el denominado mercado emergente para los profesionales de la información, en sectores como las tecnologías de la información, la investigación y los servicios de provisión y análisis de información financiera y política.

\section{«La incorporación al mundo la- boral comienza incluso antes de finalizar los estudios, princi- palmente a través de becas de trabajo»}

Los diplomados de l'École de Bibliothéconomie et des Sciences de l'Information de la Universidad de Montréal entre los años 1991 y 1995 también fueron objeto de estudio (Tabah; Bernhard, 1998). La tasa de respuesta fue del $37,5 \%$. Entre los datos más sobresalientes se puede destacar que cada graduado ocupó una media de 2,5 empleos. Los 4 ámbitos de actividad más importantes fueron en este orden: referencia, catalogación, análisis de la información y gestión. En cuanto al tiempo obtenido para encontrar el primer empleo: el $40 \%$ lo tenía ya antes de acabar los estudios; el 46,3\% tardó entre 1 y 4 meses; el 11,3\% entre 5 y 8 ; mientras que un $2,5 \%$ de 2 a 4 años. Por lo que respecta a la valoración de los campos de actividad que estos titulados hicieron, los más importantes son: consultoría, edición electrónica, vigilancia tecnológica, formación de usuarios, creación y gestión de bases de datos, asesoramiento en informática, gestión de documentos electrónicos y gestión de recursos en internet.

Quarmby, Willet y Wood (1999) llevaron a cabo el seguimiento de los graduados del programa MSc de gestión de la información de la Universidad de Sheffield entre los años 1994 y 1996, con una tasa de respuesta del $62 \%$. Como conclusiones se establece que la mayoría de los graduados obtuvo fácilmente empleo en biblioteconomía y documentación, sobre todo en los sectores industriales y comerciales, siendo las tareas más importantes la búsqueda de información, la gestión y administración así como la educación y formación. En cuanto a los componentes formativos del programa que más les han servido por importancia son los siguientes: diseño de bases de datos y análisis de sistemas, las habilidades informáticas, internet y creación de documentos html así como la destreza en la búsqueda de información. Las prácticas tradicionales de las bibliotecas son poco desarrolladas en sus empleos.

Encontramos también estudios de este tipo en el ámbito de la archivística. Los más recientes son los de Yakel (2000) y Wallace (2000). El primero analizó la inserción laboral de los graduados por los programas de nivel master en archivística en EUA. Wallace estudió en concreto la inclusión laboral de los estudiantes de los programas de archivística y records management de 10 universidades tanto de EUA como de Canadá.

Otras universidades en todo el mundo también se han preocupado por sus graduados. Citemos tan sólo algunos ejemplos: Ocholla (2000) los analizó entre 1998 y 1999 en la Universidad de Zululand en Suráfrica; Alemna (1999) a los titulados por el Departamento de Estudios de la Biblioteca y Archivos de la Universidad de Ghana entre 1992 y 1997; Aina y Moahi (1999) a los egresados del Departamento de Biblioteconomía y Documentación de la Universidad de Botswana de 1995.

\section{Estudios sobre el seguimiento de la inserción laboral de los graduados en biblioteconomía y documentación en España}

1. Estudios de seguimiento de graduados de diferentes universidades españolas.

Uno de los primeros estudios realizados fue el de Espelt y Pons (1993), quienes describieron a grandes rasgos la incorporación laboral de las promociones de 1988 a 1992 de la Escuela de Biblioteconomía Jordi Rubió i Balaguer de Barcelona, llevándose a cabo sobre una muestra de los graduados inscritos en el Col.legi Oficial de Bibliotecaris Documentalistes de Catalunya. Los datos de empleo fueron positivos en el sentido que el 96,14\% (299 diplomados de 311) habían encontrado trabajo en biblioteconomía y documentación. La mayoría en el sector público, ya que únicamente el 5,68\% se incorporó a empresas, tendencia que como veremos irá cambiando con el paso de los años. 
El primer estudio en profundidad realizado fue el de Moreiro, Moscoso y Ortiz-Repiso (1995). El colectivo objeto de análisis fue la promoción del curso 1991-1992 de las escuelas de Barcelona, Granada, Murcia y Salamanca. Se elaboró una encuesta que obtuvo una tasa de respuesta del $53,4 \%$ ya que, de un total de 525 cuestionarios enviados, fueron contestados 279. Casi la mitad de los que lo hicieron encontraron trabajo en biblioteconomía y documentación, aunque con importantes diferencias según la escuela en la que se graduaron, siendo la de Barcelona la que ofreció un dato mejor (el 75\%). La incorporación al mundo laboral comienza incluso antes de finalizar los estudios a través de las becas de trabajo principalmente. De este modo, hay un $21 \%$ que encontró ya empleo antes de acabar sus estudios. La forma de acceso fue principalmente a través de contactos y relaciones $(25 \%)$, becas (18\%), Inem (16\%), oposiciones (10\%) y envío de currícula a las empresas $(10 \%)$.

\section{«En 1996 se perfilaron algunos cambios en las tendencias de los anteriores estudios: las de- mandas del sector privado em- pezaron a crecer»}

La categoría profesional que desempeñaron fue principalmente la de ayudante por lo que, en principio, sí que hay una adecuación del nivel de estudios con la categoría profesional. El problema que se identificó es que no se correspondía luego con la relación contractual, ya que solamente el $7 \%$ de los encuestados gozaba de cierta estabilidad laboral al año de acabar la carrera, o que incluso entre un $8 \%$ y un $10 \%$ trabajaban sin ningún tipo de contrato. El sector donde más trabajo encontraron fue el público (un 79\%) y como centros, las bibliotecas fueron las principales empleadoras. Un dato preocupante fue que la opinión que tenían del prestigio social de la profesión era muy baja.

\section{Estudios de seguimiento de graduados de la Universidad Carlos III de Madrid.}

Moreiro (1998, 1999, 2000 y 2001) ha estudiando la inserción laboral de los graduados por esta universidad madrileña. Unas veces a través de los datos ofertados por su Servicio de Orientación y Planificación Profesional (en adelante Sopp) y otras mediante encuestas propias. Así, en 1998 recogía y analizaba los datos del informe del Sopp de 1996. En este año se perfilaban algunos cambios en las tendencias de los anteriores estudios: las demandas del sector privado empezaban a crecer y ya no sólo obedecían a puestos tradicionales y a bibliotecas, puesto que se constataba que el mercado en información y documentación se estaba ampliando.
En 1999 Moreiro recoge los datos del Sopp referentes a la licenciatura en documentación y a la diplomatura en biblioteconomía y documentación. Se confirma que no hay una diferenciación clara de funciones y puestos para los diplomados y licenciados por parte de las empresas. Los perfiles demandados son muy imprecisos y la oferta es muy amplia. Este mismo autor, en el año 2000 y a partir también de los datos de los informes del Sopp, sigue comprobando cómo el mercado se está ampliando fuera de las escenas tradicionales de las bibliotecas.

El último estudio de Moreiro (2001) a reseñar se publicó recientemente y presenta la evolución de la empleabilidad de los graduados en biblioteconomía y documentación en la Carlos III desde 1996 a 1999. Se aprecia un aumento considerable del empleo en estos años. Las formas de acceso han cambiado, siendo las más importantes las bolsas de empleo universitarias y los contactos personales. En cambio, la importancia del Inem y de los anuncios en prensa ha disminuido. Las becas siguen siendo determinantes e incluso son la forma que tienen las empresas para seleccionar a su personal estable. Las funciones básicas del currículum tradicional (catalogación, indización, clasificación, etc.) se siguen solicitando. Además, los empleadores dan importancia tanto a las actitudes como a las capacidades de gestión, siendo las habilidades informáticas y el inglés fundamentales.

\section{Estudios de seguimiento de graduados de la Universidad Complutense de Madrid.}

El Servicio de Información Profesional y para el Empleo (Sipe) de la Escuela Universitaria de Biblioteconomía y Documentación de la UCM (Tejada, 1999, 2000, 2001) ofrece unos resultados de su gestión que confirman la idea aportada por Moreiro (2000) referente a la creciente diversificación de la profesión. En la tabla 1 presentamos el número de ofertas, así como el sector del organismo del que proceden distribuidas en los 3 cursos académicos en que se realizaron. Como vemos, los medios de comunicación, consultoras y empresas ligadas a las nuevas tecnologías son las principales demandantes.

En enero de 2001 el Consejo Social de la UCM publicó un informe sobre la trayectoria laboral de los diplomados en biblioteconomía y documentación de esta universidad. Se analizaron las promociones comprendidas entre la 1992-93, que fue la primera, y la de 1996-97, con un universo compuesto que comprendía 540 personas. Se realizaron 260 encuestas telefónicas y la selección del individuo fue hecha mediante un muestreo aleatorio sistemático.

Entre los datos más significativos que ofrece el análisis final están los siguientes: 
—El 94\% de los entrevistados ha tenido alguna vez empleo en este sector. Hay una movilidad alta ya que la media de ocupaciones ha sido de 2,3 por persona. La precariedad es también elevada, así el $84,2 \%$ de los empleos tuvieron un carácter eventual y tan sólo el 8,1\% fue indefinido. Las becas remuneradas han ido aumentado en importancia, mientras que el $24 \%$ de los empleos de la promoción más antigua era de este tipo, en la de 1997 era del $34 \%$. Esta precariedad también ha aumentado en la administración pública.

—El 68\% de los trabajos es de carácter técnico y está relacionado con los estudios. Un 35\% corresponde al perfil de bibliotecario, un $24 \%$ de documentalista y el $2 \%$ de archivero, siendo el sector privado el que más ha empleado a los diplomados $(73,6 \%)$. La actividad principal de las empresas contra-

\begin{tabular}{|c|c|c|c|c|}
\hline Sector & $\begin{array}{l}\text { Número de ofertas } \\
\text { Curso 1998-1999 }\end{array}$ & $\begin{array}{c}\text { Número de ofertas } \\
\text { Curso } 1999-2000\end{array}$ & $\begin{array}{l}\text { Número de ofertas } \\
\text { Curso 2000-2001 }\end{array}$ & $\begin{array}{l}\text { Total } \\
\text { ofertas }\end{array}$ \\
\hline $\begin{array}{l}\text { Medios de comunicación, agencias de } \\
\text { seguimiento de prensa y agencias de } \\
\text { comunicación. Productoras TV. }\end{array}$ & 11 & 8 & 9 & 28 \\
\hline Consultoras & 4 & 6 & 5 & 15 \\
\hline Asociaciones, fundaciones y ONG & 3 & 6 & 4 & 13 \\
\hline Educación & 2 & 2 & 4 & 8 \\
\hline Informática & 3 & 2 & 3 & 8 \\
\hline Telecomunicaciones & 3 & 3 & 2 & 8 \\
\hline Empresas de internet & 1 & 2 & 4 & 7 \\
\hline Administración Pública & 2 & 2 & 3 & 7 \\
\hline Editoriales y librerias & 2 & 1 & 2 & 5 \\
\hline $\begin{array}{l}\text { Colegios profesionales y Reales } \\
\text { Academias }\end{array}$ & 2 & 2 & - & 4 \\
\hline Arquitectura e ingenieria & 3 & 1 & $\cdot$ & 4 \\
\hline Recursos humanos & - & 2 & 1 & 3 \\
\hline $\begin{array}{l}\text { Consultoras en documentación y } \\
\text { archivos }\end{array}$ & 2 & 1 & - & 3 \\
\hline Despachos de abogados & 1 & 1 & $\cdot$ & 2 \\
\hline Energia & - & - & 2 & 2 \\
\hline Investigación y estudios & 1 & - & - & 1 \\
\hline Embajadas & 1 & - & - & 1 \\
\hline Partidos politicos & - & 1 & 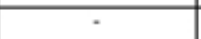 & 1 \\
\hline Industria automovilistica & - & - & 1 & 1 \\
\hline Banca & - & - & 1 & 1 \\
\hline Varios & 1 & 4 & 2 & 7 \\
\hline Total & 42 & 44 & 43 & 129 \\
\hline
\end{tabular}

Tabla 1. Número de ofertas de empleo por sector, tramitadas por el SIPE de la Escuela Universitaria de Biblioteconomía de la UCM tantes era la de servicios $(65,8 \%)$, y específicamente educación (20\%), asociaciones (13\%) y servicios a empresas (13\%). La administración pública representa un $26,4 \%$.

- La mayoría de los diplomados estaba trabajando en el momento de contestar la encuesta $(73,8 \%)$. Había también un 9,2\% que no lo hacía ni buscaba empleo ya que en su mayor parte estaban estudiando. Lo que el informe no indicaba era si trabajaban en puestos relacionados con la biblioteconomía y documentación.

- La dificultad mayor en su acceso al mundo laboral para estos diplomados es la baja valoración social de su titulación (así lo manifiesta un 46,2\%).

El último estudio que señalamos es el más reciente y constituyó una tesis doctoral (Tejada, 2002), cuyo objetivo principal fue la recolección y sistematización de las opiniones y percepciones de los diplomados en biblioteconomía y documentación por la Universidad Complutense de Madrid y de los empleadores sobre el nuevo plan de estudios ${ }^{1}$ de la Escuela Universitaria de Biblioteconomía y Documentación de la citada universidad. Además de la correspondencia entre formación y desarrollo profesional, se analizó la inserción laboral que habían tenido los diplomados entre la primera promoción del año 1993 y la del año 2000.

Para recoger los datos de su desarrollo profesional y laboral se utilizó una encuesta que se envió por correo a todos ellos. La tasa de respuesta fue del $42 \%$ ya

que contestaron 361 de un total de 855 diplomados. A continuación se ofrecen algunos de los datos más importantes recogidos en el estudio que conforman un panorama del mercado al que accede este colectivo.

En general, por el volumen de empleo este sector se puede considerar dinámico. Así, el 86,1\% de los titulados que contestaron encontró algún trabajo en este área. Estos datos apuntan un avance de la ocupación del sector frente a la situación a mediados de los 90 (Moreiro y otros, 1995), ya que no llegaba al 50\%. Esta mejoría de los resultados puede obedecer a las nuevas demandas generadas por el impulso de internet y por la apertura de nuevos nichos de trabajo, tal como reflejan los datos de la propia bolsa de trabajo de la Escuela de Biblioteconomía y Documentación de la UCM.

La fácil inserción laboral también lo señala el tiempo transcurrido hasta obtener el primer empleo en relación con su formación académica. Así el porcentaje de estos diplomados que tardaron menos de un año en obtener un trabajo referente a su titulación supone un $71,5 \%$. Incluso un $41 \%$ ya lo obtuvo antes de finalizar sus estudios.

Además, el desempleo tampoco es preocupante en cuanto al tiempo máximo en el que están en esta situación: el 25,5\% afirma no haberlo estado nunca y los 
que esperaron más de un año únicamente fue el 14,7\% del total.

En el cuestionario utilizado, a diferencia del de otros estudios, se pedía que señalaran las características de todos las ocupaciones en biblioteconomía y documentación que habían tenido. Un primer rasgo que destaca es el número de empleos que han ocupado: 855 , lo que supone que como media tenemos 2,8 por persona, ya que fueron 311 diplomados los que obtuvieron trabajo. Este hecho tiene una doble lectura: positiva, puesto que indica el ya señalado dinamismo, y negativa, en cuanto que también apunta una excesiva movilidad por el carácter temporal de estas ocupaciones.

Cada uno de estos empleos fue descrito según las características del puesto y del tipo de centro en que se desarrolló la colocación; sector al que pertenece; forma de acceso; factores que han influido en su obtención; tipo de relación contractual; duración; y remuneración.

\section{«La universidad debe estudiar la inserción laboral y el desarro- Ilo profesional de sus gradua- dos para así poder adecuar me- jor sus programas académicos»}

La mayoría obtuvo un puesto en un nivel relacionado con su titulación. Así, los que ocuparon un nivel de auxiliar representaron sólo el 33,5\% del total, aunque este dato luego no se corresponde con los salarios o en las condiciones. De esta forma, el $38,8 \%$ de los puestos obtenidos fueron becas y el $39 \%$ contratos temporales. En cuanto a la temporalidad, el $37,4 \%$ de estos empleos tuvo una duración menor de 6 meses. Además, el 83\% tuvo una renumeración inferior a los 900 EUR (150.000 PTA) netos mensuales. Todo esto indica precariedad del mercado al que acceden estos titulados.

En lo referente al tipo de centro, nos encontramos que la biblioteca sigue siendo aquel en el que más cabida tienen los diplomados (43\% de los puestos), aunque el porcentaje de centros de documentación es también muy alto (39\%) y aumenta con respecto al porcentaje del estudio de Moreiro y otros (1995).

Sobre el sector señalar que es en el público donde han encontrado más trabajo los diplomados: $47,7 \%$ del total frente al 39\% del sector privado y del 13\% de otros. Este dato representó un cambio muy importante de tendencia sobre lo hallado por Moreiro y otros (1995), según el cual el 79\% del empleo pertenecía al sector público. Este hecho también es señalado en el estudio de Moreiro (2001) sobre la inserción laboral de los licenciados en documentación por la Universidad Carlos III de Madrid, que presenta unas conclusiones similares: $51,5 \%$ de empleos para el sector público y $48,4 \%$ para el privado.

En el caso de este último, los principales empleadores de los titulados que respondieron a la encuesta son en este orden: los medios de comunicación, al igual que las empresas en general constituyen $89 \mathrm{em}$ pleos (10\% del total), el sector informático y de telecomunicaciones con $72(8,1 \%)$, las editoriales con 34 $(3,8 \%)$, la consultoría con $32(3,6 \%)$ y las fundaciones y ONGs con $29(3,2 \%)$.

\section{Conclusiones}

La universidad, responsable de la formación de profesionales, debe estudiar la inserción laboral y el desarrollo profesional de sus graduados para así poder adecuar mejor sus programas académicos.

La metodología de los estudios realizados se ha basado en encuestas como forma de obtener los datos referentes al empleo al que acceden los titulados.

Estos estudios tienen un alto componente ocupacional que se ve ensombrecido por las características precarias de los trabajos desarrollados. Estas malas condiciones se manifiestan en figuras contractuales muy poco estables, con un predomino de las becas, en una duración de los empleos muy reducida y en unos sueldos que no se corresponden con el nivel de la ocupación.

En cuanto al tipo de institución y sector al que pertenece hay que destacar que el trabajo en centros de documentación en el sector privado ha aumentado porcentualmente más que en bibliotecas y archivos del sector público. Los escenarios tradicionales de la profesión de principios y mediados de la década de los 90 se han ampliado y diversificado en gran medida.

\section{Nota}

1. Entró en vigor en el curso 2000-2001. Publicado en el BOE el 5 de julio de 2000 .

\section{Bibliografía}

Abadal Falgueras, E.; Miralpeix, C. "La enseñanza de la biblioteconomía y documentación en la universidad española a finales de los noventa". En: BiD: textos universitaris de biblioteconomia i documentació, 1999, març, n. 2. Consultado el: 08-11-02.

http://www.ub.es/biblio/bid/02abamir.htm

Aina, L. O.; Moahi, K. "Tracer study of the Botswana library school graduates". En: Education for information, 1999, v. 17, pp. 215-244.

Alemna, A. A. "Career development: follow-up studies of former graduate students of the Department of library and archival studies University of Ghana, 1991/1992 to 1996/1997". En: Education for information, 1999, v. 17, n. 1, pp. 35-43. 
Aslan, G. "L'avenir des diplômés en bibliothéconomie et sciences de l'information: analyse de la concurrence". En: Argus, v. 26, n. 1, printempsété 1997, pp. 11-20.

Crane, H. "New librarians: recent graduates on library studies and work". En: Feliciter, 1998, September, v. 44, n. 9, pp. 28-31.

Deschamps, J. Nouvelles competences en information documentaire et technologie de l'information: étude des offres d'emploi du Bulletin des postes vacants de l'Esid sur quatre années de référence. 1999. Consultado el: 26-12-01.

http://www.geneve.ch/heg/id/presentation/missions/urtexte2.htm

Espelt, C.; Pons, A. "Dades sobre la incorporació laboral de les darreres promocions de diplomats de l'Escola Jordi Rubió i Balaguer". En: Item, 1993, n. 13 , pp. $95-103$

Marcella, R.; Baxter, G. A. "Career progression survey of graduates of the Postgraduate Diploma/MSc in information analysis". En: Education for information, 1998, n. 16, pp. 107-130.

Mayol, C. "La formació del professionals". En: BiD: textos universitaris de biblioteconomia i documentació, 2000, juny, n. 4. Consultado el: 0811-2002.

http://www.ub.es/biblio/bid/04mayol.htm

Moreiro González, J. A.; Moscoso, P.; Ortiz-Repiso, V. "El mercado de trabajo de los diplomados españoles en biblioteconomía y documentación". En: Revista española de documentación científica, 1995, v. 18, n. 4, pp. 444-463.

Moreiro González, J. A. "Mercado de trabajo y competencias profesionales en biblioteconomía y documentación: técnicas aplicables a su investigación". En: Informação e sociedade, 1998, v. 8, n. 1, pp. 124-151.

Moreiro González, J. A.; Caridad, M. "Acerca de los métodos de estudio de la relación entre las condiciones laborales y formativas en biblioteconomía y documentación: el caso de la Universidad Carlos III". En: Anales de documentación, 1998, n. 1, pp. 137-153.

Moreiro González, J. A. "Ajuste de los licenciados en documentación a la oferta de trabajo. Observaciones hechas en la Universidad Carlos III de Madrid". En: Revista general de información y documentación, 1999, v. 9 , n. 2, pp. 13-28.

Moreiro González, J. A. "Algunas características de la formación de los documentalistas en el entorno cambiante de la sociedad y la economía de la información 2000". En: IX Jornadas Eubd: documentación. Los profesionales ante el nuevo milenio, 2000. Consultado el: 05-07-01.

http://www.eubd.ucm.es/actividades/jornadas/moreiro/JornadasComplute nse2000.rtf

Moreiro González, J. A. "Licenciatura en documentación: experiencias, desarrollo y problemática. El caso de la Universidad Carlos III de Madrid". En: Anales de documentación, 2001, n. 4, pp. 151-168.
Moreiro González, J. A. "Figures on employability of Spanish library and information science graduates". En: Libri, 2001, v. 51, pp. 27-37.

Ocholla, D. N. "Review and revision and information science curriculum in a South African University and the usage of follow-up study and advertisement scanning methods". En: Cais 2000: dimensions of a global information science, 2000, Edmonton. Consultado el: 08-11-02. http://www.slis.ualberta.ca/cais2000/ocholla.htm

Quarmby, K. L.; Willett, P.; Wood, F. E. "Follow-up study of graduates from the MSc information management programme at the University of Sheffield". En: Journal of information science, 1999, v. 25. n. 2, pp. $147-$ 155.

Tabah, A. N.; Bernhard, P. "Emplois occupés après la diplômation et champs d'activité perçus comme importants "aujoud'hui" et "dans cinq ans" selon l'enquête menée auprès des diplômes de l'Ebsi (1991-1995)". En: Argus, 1998, automme, v. 27. n. 2, pp. 5-13.

Tejada Artigas, C. Adecuación de los planes de estudio de la diplomatura en biblioteconomía y documentación a las demandas del mercado de trabajo. El caso concreto del nuevo plan de estudios de la Escuela Universitaria de Biblioteconomía y Documentación de la Universidad Complutense de Madrid. Tesis doctoral. Madrid: Universidad Carlos III, 2002.

Tejada Artigas, C. Informe de las actividades desarrolladas por el Sipe durante el curso 2000-2001. Madrid, 2001.

Tejada, C. Informe de las actividades desarrolladas por el Sipe durante el curso 1998-99. Madrid, 1999.

Tejada, C. Informe de las actividades desarrolladas por el Sipe durante el curso 1999-2000. Madrid, 2000.

Trayectorias laborales de los diplomados en biblioteconomía y documentación. Madrid: Consejo Social. Universidad Complutense de Madrid, 2001.

Wallace, D. A. "Survey of archives and records management graduate students at ten universities in the United States and Canada". En: The American archivist, 2000, fall/winter, v. 63, pp. 284-300.

Yakel, E. "The future of the past: a survey of graduates of master's-level archival education programs in the United States". En: The American archivist, 2000, fall/winter, v. 63, pp. 301-321.

Carlos Tejada Artigas, Escuela Universitaria de Biblioteconomía y Documentación de la Universidad Complutense de Madrid.

tejada@caelo.eubd.ucm.es

José Antonio Moreiro, Facultad de Humanidades Comunicación y Documentación de la Universidad Carlos III de Madrid.

jamore@bib.uc3m.es

\title{
Próximos números monográficos
}

\author{
Marzo 2003 Estándares documentales en internet: Z39.50, Dublin Core... \\ Mayo $2003 \quad$ Auditoría de la información \\ Julio 2003 Administración electrónica (e-government)
}

Los interesados pueden remitir notas, artículos, propuestas, publicidad, comentarios, etc., sobre estos temas a:

epi@sarenet.es 